\title{
The Evolution Sequence in Narrow-Line and Broad-Line SEYFERT 1 Galaxies
}

\author{
Y. Z. Wu ${ }^{1}$ and S. N. Zhang ${ }^{1,2}$ \\ ${ }^{1}$ Institute of High Energy Physics, Chinese Academy of Sciences, \\ email: yzwu@ihep.ac.cn \\ ${ }^{2}$ National Astronomical Observatories, Chinese Academy of Sciences, \\ email: zhangsn@ihep.ac.cn
}

\begin{abstract}
Collecting a sample of 50 narrow-line Seyfert 1 galaxies (NLS1s) and 44 broadline Seyfert 1 galaxies (BLS1s), we show that the theoretical maximum starburst line derived by Kewley et al.can separate well BLS1s from NSL1s on the BPT diagrams, and the [N II] $\lambda 6584 / \mathrm{H} \alpha$ ratio increases along the list from NLS1s to BLS1s. We suggest that: (1) they have a sequence from low metallicities to high metallicities between NLS1s and BLS1s; (2) with regard to the "star-froming sequence" of Kewley et al.who noted that it forms a tight sequence from low metallicities to high metallicities, the sequence may be connected to the NLS1-BLS1 sequence of "active galactic nucleus (AGN)" sequences.
\end{abstract}

Keywords. Galaxies: active — galaxies: Seyfert — galaxies: statistics.

\section{Introduction}

According to the unified model of AGNs, Seyfert 1 galaxies (Sy1s) are seen face-on and have both narrow and broad emission lines; Sy2s are seen edge-on and have only narrow emission lines, which are commonly believed to be intrinsically the same as type 1 AGN (Antonucci 1993; Wu et al. 2011). Sy1s are usually classified into two subgroups based on the full width at half-maximum (FWHM, larger or smaller than $2000 \mathrm{~km} \mathrm{~s}^{-1}$ ) of their broad $\mathrm{H} \beta$ lines. Observational evidence shows that NLS1s tend to have small black hole masses and high Eddington ratios (Komossa \& Xu 2007; Zhu, Zhang, \& Tang 2009).

Considering that NLS1s do not follow the $M_{\mathrm{BH}}-\sigma_{*}$ relation and accretion rates in NLS1s are higher than those in BLS1s, a conclusion that NLS1s are young AGNs is drawn naturally (Mathur 2000). As black holes of NLS1s grow, they will get closer to the $M_{\mathrm{BH}}-\sigma_{*}$ relation (Grupe \& Mathur 2004). The BPT diagram is one of the best distinguishable means for star-forming galaxies (SFs) and AGNs, and it also displays well the evolutionary relation in SFs (Kewley et al. 2006; Yuan et al. 2010). In Wu, Zhao, \& Meng (2011), they demonstrate the evolution sequence from starburst galaxies to non-hidden broad-line region (non-HBLR) Sy2s and then to HBLR Sy2s with the BPT diagram. Here, we investigate the evolution between NLS1s and BLS1s with the diagram.

\section{Overview}

The sample is compiled from the catalogue of active nuclei in Véron-Cetty \& Véron (2001) and Zhou et al. (2006). The BLS1 sample is selected from the catalogue of VéronCetty \& Véron (2001), including four subgroups: Seyfert 1 (S1), S1.0, S1.2, and S1.5, and having 2421 Sy1 galaxies. In Véron-Cetty \& Véron (2001) and Zhou et al. (2006), the subgroup "S1n" and the NLS1 samples, which have 150 and 2011 NLS1 galaxies, respectively, are chosen initially the NLS1 sample. The above NLS1 and BLS1 samples 


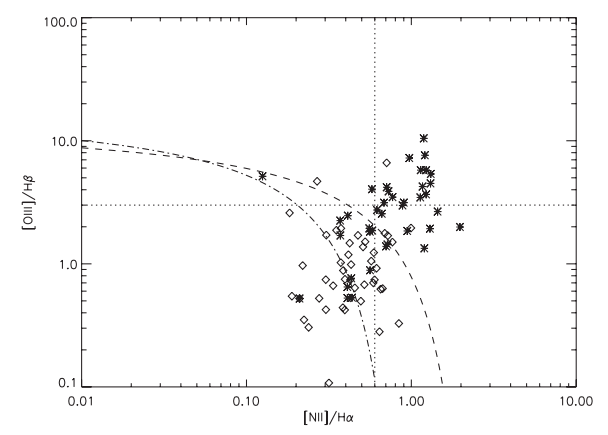

Figure 1. [N II] $\lambda 6584 / \mathrm{H} \alpha$ versus [O III] $\lambda 5007 / \mathrm{H} \beta$ for NLS1s and BLS1s. The open diamond and asterisk denote NLS1s and BLS1s, respectively. The dashed curve is the theoretical maximum starburst line derived by Kewley et al. 2001 as an upper limit for SFs; the dot-dashed curve on the diagram is the Kauffmann et al. (2003) semiempirical lower boundary for the SFs.

are cross-matched by the catalogue of the MPA-JHU DR7 release. Finally, we obtain the sample of 44 BLS1s and 50 NLS1s.

In Figure 1, we show the distribution of $[\mathrm{N} \mathrm{II}] / \mathrm{H} \alpha$ versus $[\mathrm{O} \mathrm{III]} / \mathrm{H} \beta$ for NLS1s and BLS1s. In the diagram, BLS1s are preferentially in the upper right corner while NLS1s have the trend of residing in the lower left corner. The theoretical maximum starburst line $(\log ([\mathrm{O}$ III $] \lambda 5007 / \mathrm{H} \beta)=0.61 /(\log ([\mathrm{N} \mathrm{II}] / \mathrm{H} \alpha-0.47)+1.19))$ derived by Kewley et al. (2001) is used to define as the separation of BLS1s and NSL1s: BLS1s mainly display a left boundary (24/39) while NLS1s mostly display a right boundary $(38 / 44)$. The means of $[\mathrm{N} \mathrm{II}] / \mathrm{H} \alpha$ ratios for NLS1s and BLS1s are $0.462 \pm 0.028$ and $0.824 \pm 0.063$, respectively.

According to the "star-forming sequence" of Kewley et al. (2006) who noted that it forms a tight sequence from low metallicities (low $[\mathrm{N} \mathrm{II}] / \mathrm{H} \alpha$, high $[\mathrm{O} \mathrm{III}] / \mathrm{H} \beta$ ) to high metallicities (high $[\mathrm{N} \mathrm{II}] / \mathrm{H} \alpha$, low $[\mathrm{O} \mathrm{III}] / \mathrm{H} \beta$ ), and the sequence is connected to the beginning of the "mixing sequence of AGN and star formation" (Kewley et al. 2006; Yuan et al. 2010). Finally, they may be extended toward high $[\mathrm{N} \mathrm{II}] / \mathrm{H} \alpha$ and high $[\mathrm{O}$ III $] / \mathrm{H} \beta$ values, which may be regarded as an "AGN sequence". In Wu, Zhao, \& Meng (2011), since the $[\mathrm{N} \mathrm{II}] / \mathrm{H} \alpha$ ratios increase constantly along the list from starburst galaxies to non-HBLR Sy2s and then to HBLS Sy2s, they suggested an evolutionary sequence from starburst galaxies to non-HBLR Sy2s and then to HBLS Sy2s. Therefore, these show that the evolutionary relation was demonstrated by the $[\mathrm{N} \mathrm{II}] / \mathrm{H} \alpha$ ratio in $\mathrm{SFs}$, and the ratio displays that in other galaxies.

\section{References}

Antonucci, R. 1993, ARAA, 31, 473

Grupe, D. \& Mathur, S. 2004, ApJ, 606, L41

Kauffmann, G., et al. 2003, MNRAS, 346, 1055

Kewley, L. J., et al. 2001, ApJ, 556, 121

Kewley, L. J., Groves, B., Kauffmann, G., \& Heckman, T. 2006, MNRAS, 372, 961

Komossa, S. \& Xu, D. 2007, ApJ, 667, L33

Mathur, S. 2000, MNRAS, 314, L17

Wu, Y. Z., Zhang, E. P., Liang, Y. C., Zhang, C. M., \& Zhao, Y. H. 2011, ApJ, 730, 121

Wu, Y. Z., Zhao, Y. H., \& Meng, X. M. 2011, ApJS, 195, 17

Yuan, T. T., Kewley, L. J., \& Sanders, D. B. 2010, ApJ, 709, 884

Zhou, H. Y., et al. 2006, ApJS, 166, 128

Zhu, L., Zhang, S. N., \& Tang, S. M. 2009, ApJ, 700, 1173 